\title{
Ueber \\ die Endigung der Nerven im quergestreiften Muskel der Wirbelthiere.
}

Von

E. Fischer, stud, rer. nat.

(Aus dem histologischen Laboratorium von Prof. Kollmann in München.)

Hierzu Tafel XXV und XXVI.

In Bezug auf die Endigung der Nerven im willkürlichen Muskel hat vor Kurzem Gerlach ${ }^{1}$ ) die Ansicht aufgestellt, dass die Nervenfasern nicht, wie man früher annahm, mit motorischen Endplatten endigen, sondern dass sie in netzförmig zusammenhängende, feine Fasern übergehen, welche im Inneren der Muskelfäden, der ganzen Länge derselben nach, verlaufen und hiebei mit den isotropen Elementen der contractilen Substanz wahrscheinlich in Verbindung treten (intravaginale Nervennetze).

Nun habe ich bei Gelegenheit von Untersuchungen, die ich über Nervenendigungen in der Haut und zwar beim Meerschweinchen anstellte, das Vorhandensein von Endplatten an den Muskelfäden bestätigt gefunden und habe darauf hin eine Untersuchung der Nervenendigung im quergestreiften Muskel zuerst bei verschiedenen Säugethieren, dann in absteigender Reihe bei den übrigen Klassen der Wirbelthiere vorgenommen, deren Resultate ich in Folgendem kurz mittheilen will.

1) Sitzgsber. d. phys.-med. Societät zu Erlangen. Jahrg. 1873. Heft V. pag. 97. - Das Verhältniss d. Nerven z. d. willkürl. Muskeln der Wirbelthiere. Leipzig 1874. 
Von Säugethieren habe ich Meerschweinchen, Kaninchen, Hund, Katze, Schwein und den Menschen untersucht. Zur Untersuchung verwandte ich Hautmuskeln, vom Menschen den M. orbicularis oris, vom Schwein die im subcutanen Gewebe und der Cutis der vorderen Fläche des Rüssels gelegenen Muskeln, von den übrigen der genannten Thiere die Muskeln in der Haut der Wangengegend. Durch diese Verwendung von Hautmuskeln wurde ich in die Lage versetzt, bei Anwendung der von Löwit'schen Goldmethode, deren ich mich zur Darstellung der Nervenfasern und ihrer Endigung in erster Linie bediente, genau in der Weise vorzugehen, wie ich von dem erwähnten Verfahren, einer mündlichen Mittheilung Löwit's folgend, schon behufs Darstellung des Nervenfaserverlaufs in den Tastkörpern des Menschen Gebrauch gemacht hatte. Ich kann demnach bezüglich der Methodik auf die Abhandlung von Löwit ${ }^{1}$ ) und auf meine Arbeit über die Tastkörper ${ }^{2}$ ) verweisen.

An Schnitten der nach dem Löwit'schen Verfahren vergoldeten Hautstückchen fand ich die Muskelfasern der genannten Säugethiere in den Fällen, in welchen mir die Darstellung von Endplatten gelungen war, entweder farblos oder in verschiedenen Tönen diffus gefärbt. Distinkte mit Gold gefärbte Längsstreifen oder längs verlaufende und netzförmig zusammenhängende feine Fasern waren in diesen Fällen an denselben nicht wahrzunehmen. Dagegen war die Längs- und Querstreifung an den farblosen Muskelfäden häufig noch deutlich ausgeprägt. - Das Verhalten der zutretenden Nervenfasern an den Muskelfäden ist nach dem Effekt der Vergoldung folgendes: An einer, häufig, jedoch nicht immer (s. die Figg. $3 \mathrm{~A}, 4 \mathrm{~A}$ u. B), hügelartig erhobenen Stelle (s. d. Figg. 7 u. 10) der Muskelfasern angekommen, gehen die beim Zutritt durch das Aufhören der Markscheide sich plötzlich verdünnenden Nervenfasern in eigenthümlich verbreiterte Fasern über, welche, während sie sich mannigfach verzweigen und gegen einander biegen, einen abgegrenzten Bezirk der Oberfläche der Muskelfasern inne halten und auf diese Weise plattenähnliche Gebilde formiren (s. d. Figg. 1, 2, 5, 6, 8 und 11). Die Endigung dieser die Endplatten bildenden Fasern geschieht nach Goldpräparaten meistens mit einer verschieden geformten Verdickung

1) Wien. Sitzgsber. Bd. LXXI. Abth. III. 1875. pag. 1.

2) Dieses Archiv Bd. 12. pag. 366. 
(s. d. Figg. 1-9 und 11 bei a), manchmal aber enden die Fasern auch nur zugerundet (s. d. Figg. 1 und 7 bei b) oder zugespitzt (Fig. 2, 5, 6 bei c). Verdickungen von ähnlicher Form, wie diese die Fasern abschliessenden, finden sich auch, und zwar nicht selten, in dem Verlauf der Fasern eingeschaltet (s. d. Fjgg. 1-6, 8 und 11 bei d). Aehnlich geformte Gebilde kommen ferner häufig auch isolirt in den Endplatten vor (s. d. Figg. 6, 7, 10 und 11 bei e), und sind diese Fälle zurückzuführen entweder auf den von Kü $_{\text {un }} \mathrm{e}^{1}$ ) beschriebenen Zerfall der Endplatten oder vielleicht auch darauf, dass feine Fasern, welche diese Verdickungen mit anderen verbunden hatten (wie sie in Fig. 11 bei f noch vorhanden sind), durch die Goldbehandlung nicht gefärbt worden sind. Wie die Figuren 1-11 lehren, zeigen sich die Fasern der Endplatten scharf begrenzt und sind weder an dem Ende noch an der den Muskelfasern zugekehrten Fläche (s. d. Profilbilder 4, 7 und 10) Andeutungen einer Fortsetzung derselben in feinere, nach dem Innern der Muskelfäden ausstrahlende Fasern vorhanden. Nur in einzelnen Fällen, deren einen ich in Fig. 2 abgebildet habe, ging eine Faser von der Platte weiter, trat aber, an der Oberfläche der Muskelfaser sich haltend, zu ähnlichen goldgefärbten Verdickungen, wie sie innerhalb der Endplatten sich so häufig finden.

Vergleicht man nun die gegebene Beschreibung und die gezeichneten durch Gold dargestellten Endplatten mit den Angaben und Abbildungen von $\mathrm{Kühne,} \mathrm{so} \mathrm{ergiebt} \mathrm{sich,} \mathrm{dass} \mathrm{die} \mathrm{Richtigkeit}$ der letzteren auf das Vollkommenste bestätigt wird. Es zeigt nämlich dieser Vergleich, dass gerade das Hauptmoment der $K \ddot{u} h n e^{\prime}$ 'schen Auffassung des Baues der Endplatten durch die Vergoldung seine Bestätigung findet, dass nämlich die eigentliche Endplatte, d. h. der äussere homogene Theil des Nervenhügels von Külne, eine Ausbreitung des Axencylinders selbst darstellt, gebildet durch eine flach ausgebreitete Verzweigung ${ }^{2}$ ). Was den inneren Theil des Nervenhügels, die Sohle fein granulirter Substanz, betrifft, welche Kü hne als Unterlage der nervösen Ausbreitung beschreibt, so ist über diese nach Goldpräparaten nichts Sicheres zu ermitteln und kann ich nur angeben, dass ein feinkörniger Goldniederschlag in vielen Endplatten, bei Profilbildern (Fig. 4, 7 und 10) unter, bei Flächenbildern (Fig. 1,

1) Strickers Handb. d. Gewebelehre. pag. 159.

2) 1. c. p. 165 . 
2, 5, 6 und 8) zwischen den vergoldeten Nervenfasern sich findet, welcher vielleicht als eine durch die Goldbehandlung hervorgerufene Färbung der Plattensohle zu betrachten ist. Immer aber ist hierbei zu bedenken, dass dieser Niederschlag in vielen der vergoldeten Endplatten (s. d. Figg. 3, 9 und 11) fehlt. Gegen die contractile Substanz scheint sich der erwähnte Niederschlag mit einer ziemlich scharfen Grenze abzusetzen (s. d. Figg. 4, 7 und 10); Beziehungen der die Endplatten bildenden Fasern zu dem vielleicht als gefärbte Plattensohle $z u$ betrachtenden Niederschlag waren an den Goldpräparațen nie zu ermitteln.

Das Vorkommen getrennter Terminalfasern in den Endplatten und die beschriebene Endigung derselben in Anschwellungen findet sich bei $K u ̈ h n$ ne nicht besonders erwähnt, dagegen sind diese beiden Momente schon durch W. Krause ${ }^{1}$ ) hervorgehoben worden.

Bei den Vögeln, von denen ich die Ente und Taube untersuchte, habe ich ebenfalls Endplatten darstellen können. Zur Untersuchung verwandte ich hier den M. complexus, den ich auf folgende Weise behandelte. Der abgetrennte Muskel wurde der Länge nach in ca. 1-2 Mm. dicke Scheiben zerlegt, diese wieder in kürzere ca $10 \mathrm{Mm}$. lange Stückchen zerschnitten und diese Stückchen mit verdünnter Ameisensäure (Säure von 1,06 spec. Gew. 1 Thl. zu 2 Thln. aqu. dest.) behandelt, bis sie durchsichtig wurden. Während die Muskelstückchen sich in der Ameisensäure befanden, wurden sie mit Nadeln möglichst auseinandergezerrt, damit die Goldlösung leichter eindringe; in diese (Goldchlorid 1:100) wurden die Stückchen direkt aus der Ameisensäure gebracht und blieben darin eine Viertelstunde. Nachdem die Muskelstückchen dann mit aqu. dest. abgewaschen worden waren, legte ich sie, nach der von Löwit beschriebenen Methode, in eine Lösung von Ameisensäure von $1: 3$ Thln. aqu. dest., in welcher sie 24 Stunden blieben. Die von Löwit angegebene Nachbehandlung mit der unverdünnten Säure liess ich weg. - Die vergoldeten Endplatten der Vögel nun sind besonders dadurch ausgezeichnet, dass in ihnen sehr häufig und zahlreich isolirte Ver-

1) S. Krause. Motor. Endplatten. Hannov. 1869. pag. 192. - Zeitschr. f. Biologie 1869. Bd. V. pag. 425 und: Handb. d. menschl. Anatomie v. C. Fr. Th. Krause. Hannover 1876. Bd. I. pag. 491. 
dickungen sich finden (s. d. Figg. 12-14). Indem nun aber nach der Aneinanderreichung dieser Gebilde (sei es, lass in diesen Fällen Fasern in kleine Theile zerfallen sind oder dass feine die Verdickungen unter einander verbindende Fasern bei der Vergoldung sich nicht gefärbt haben) ein Zusammenhang derselben unter einander wohl anzunehmen sein dürfte, stellt sich der Bau der Endplatten bei den Vögeln in ganz analoger Weise dar, wie bei den Säugethieren, nämlich in der Weise, dass eine faserartige Fortsetzung des Axencylinders durch Verzweigung und Gegeneinanderbiegen der Theilungsfasern Platten bildet. Manchmal aber (s. d. Fig. 14) krümmen die Fasern sich nicht gegen einander, sondern verlaufen nur parallel neben einander, bilden also keine Endplatten im eigentlichen Sinne. Der Uebergang der Endplatten in die gestreckte Faserausbreitung scheint mir durch die Reihenfolge der Figg. 12-14 ziemlich gut illustrirt zu werden. Was die Endigung der die Endplatten bildenden Fasern bei den Vögeln anbelangt, so ist es in Endplatten, wie Fig. 12 eine darstellt, allerdings nicht möglich, die die Fasern abschliessenden Verdickungen genau z.u erkennen, doch treten auch in solchen Fällen an einzelnen Stellen (wie in Fig, 12 bei a) Endanschwellungen hervor und sind solche in anderen Endplatten, wie in den Figg. 13 und 14 bei a, vollkommen deutlich ausgeprägt. Es enden somit auch bei den Vögeln die die motorische Nervenendigung darstellenden Fasern in Verdickungen oder Anschwellungen. $\mathrm{Zu}$ bemerken habe ich noch, dass an der Nervenendigungs-Stelle bei den Vögeln keine ausgeprägte, hügelartige Erhebung der Muskelfasern sich vorfand und dass der bei den Endplatten der Säugethiere beschriebene, zwischen den Nervenfasern befindliche Goldniederschlag bei den beobachteten Endplatten von Vögeln fehlte.

Was noch das Verhalten der Muskelfäden der Vögel nach der Vergoldung betrifft, so zeigen sich dieselben häufig von deutlichen goldgefärbten Längsstreifen durchzogen, die eine hellere Farbe besitzen, als die Substanz der vergoldeten Endplatten, und bei Betrachtung mit starken Vergrösserungen aus aneinander gereihten, kleinen Kügelchen ähnlichen Gebilden bestehen, wodurch sie ein rosenkranzähnliches oder gekerbtes Ansehen gewinnen (s. d. Fig. 12). Diese goldgefärbten Längslinen waren jedoch nicht an allen vergoldeten Muskelfäden wahrzunehmen, an welchen Endplatten zur Darstellung gelangt waren, sondern statt derselben fanden sich häufig, wenn die Muskelfäden nämlich farblos geworden waren, farblose Quer- und 
Längsstreifen (s. die Figg. 13 u. 14). Es ergiebt sich demnach ein Unterschied in dem Verhalten der Längsstreifen und der die Endplatten bildenden Fasern gegen Gold. Fin Zusammenhang der gefärbten Längsstreifen mit der Substanz der Endplatten konnte in keinem Falle nachgewiesen werden.

Bei Vergleich der bei den Vögeln sich findenden Endplatten mit der Anschauung von $K \ddot{u} h n$ e ergibt sich, dass sie derselben vollkommen entsprechen. Auf das in Fig. 14 dargestellte Verhältniss werde ich später zurückkommen.

Den Endplatten der Säugethiere sehr ähnlich sind die Endplatten der Reptilien, von denen ich nur Lacerta viridis unterscheiden konnte. Ich vergoldete nach dem Löwit'schen Verfahren die Muskeln des Oberschenkels und Oberarms dieses Thieres und verfuhr hiebei ganz nach der bei den Vögeln beschriebenen Weise. Wie bei den Säugern bestehen die Endplatten aus einer verzweigten Ausbreitung des Axencylinders, deren Fasern sich gegen einander biegen und mit verschieden geformten Verdickungen enden (s. die Fig. 15). Es ergibt sich demnach auch für die Reptilien eine vollkommene Bestätigung der Kühne'schen Befunde. Dagegen konnte ein Uebergang der Endplatten-Fasern in die auch an den Muskelfäden der Eidechse sich findenden goldgefärbten Längsstreifen nicht nachgewiesen werden (s. die Fig. 15). 'Diese Längsstreifen hatten bei der Behandlung mit Gold eive vollkommen andere, hellere Nuance angenommen, als die Fasern der Endplatten und zeigten eine ungemein deutliche, rosenkranzartige Beschaffenheit, indem sie aus in der Längsrichtung an einander gereihten Körperchen von ungefähr rundlicher Gestalt bestanden, die meistens völlig isolirt hinter einander lagen (s. die Fig. 17), manchmal jedoch, an einer Schnur aufgezogenen Perlen ähnlich, gleichsam lokale Verdickungen eines Fadens darstellten (Fig. 15 bei a). Indem nun diese Körperchen gleichsam durch überspringende Linien mit einander verbunden werden (s. die Fig. 15), entstanden Querstreifen der Muskelfäden und zwar fanden sich bald diese Querstreifen, bald die erwähnten Längsstreifen an den einzelnen Muskelfäden überwiegend entwickelt. Durch genauere Einstellung konnte ich in einzelnen Fällen, besonders an recht dünnen Stückchen der Muskelfäden, mich auch davon überzeugen, dass die genannten, überspringenden Linien nur scheinbar waren und nur 
einer nahen Aneinanderlagerung der erwähnten kleinen Körperchen ihr Hervortreten verdankten, welche in Wirklichkeit auch in der Querrichtung isolirt neben einander lagen (s. Fig. 17). Wie bei den Vögeln, so gelangten auch bei den Reptilien die goldgefärbten Längsstreifen häufig nicht zur Darstellung in Fällen, in dlenen die Endplatten sich aufs Deutlichste gefürbt hatten (s. Fig. 16). Nachzutragen habe ich noch, dass an den vergoldeten Muskelfäden der Eidechse deutliche Nervenhügel sich finden (s. Fig. 16) und dass, wie bei den Säugethieren, meistens ein feinkörniger Goldniederschlag zwischen den Endplatten-Fasern vorhanden ist, der meistens sogar sehr stark ausgeprägt sich vorfindet). Fig 15 und 16).

Ich gehe jetzt zu den Amphibien über, von denen ich nur den Frosch untersucht habe. Mein Verfahren betreffend, so verwandte ich zur Darstellung der Nervenendigung mittelst der Löwit'schen Methode die Muskeln an der Beugeseite des Oberschenkels, nachdem ich am Gastrocnemius einige resultatlose Versuche gemacht. Vor der Einlegung in die Goldchloridlösung liess ich die Muskelstückchen, da ich mit der Quellung in verdünnter Ameisensäure längere Zeit keine günstigen Resultate erlangte, in verdünnter Essigsäure quellen, verfuhr aber dann ganz, wie ich es im Absatz über die Nervenendigung bei den Vögeln beschrieben. Was nun die Nervenendigurg an den Muskelfasern des Frosches betrifft, so wurde bei diesem als terminale Ausbreitung der Nervenfasern bekanntlich von $\mathrm{K} u ̈ h n \mathrm{e}^{1}$ ) ein System sich verzweigender Fasern beschrieben, die in gestrecktem Verlaufe parallel der Längsaxe der Muskelfäden hinziehen und endlich zugerundet oder zugespitzt enden, vor dem Ende jedoch manchmal eigenthümliche Körper zeigen, die Kühne zur Endigung in Beziehung bringt und als Endknospen bezeichnet. Von anderen Autoren, wie Kölliker $\mathbf{r}^{2}$ ), Krause ${ }^{3}$ ), Engelmann ${ }^{4}$ ) u. A. wurden diese Körper jedoch als Kerne und zwar als Kerne des Neu-

1) W. Kühne, Comptes rendues pag. 316. 1861. 18 Fév. und: Ueber d. peripher. Endorgane d. motor. Nerven. Leipz. 1862.

2) Untersuchungen über d. letzt. Endigungen d. Nerven. I. Abhandlung. pag. 8.

3) Zeitschr. f. rat. Medizin. Bd. 21. pag. 77. pag. 21.

4) Untersuchungen üb. d. Zushg. v. Nerv- u. Muskelfaser. Leipz. 1863. 
rilemms erkannt. Die Angaben von Kühne in Betreff der sogenannten Endknospen konnte ich anch nicht bestätigen, sondern fand, wie die vorher genannten Autoren, einfache, den Nervenfasern direkt anliegende Kerne, wie ich sie in Fig. 19 bei a von der Fläche, bei b von der Seite gesehen abgebildet habe. Die übrigen Verhältnisse der Nervenendigung, wie sie nach Anwendung des Löwit'schen Verfahrens sich darstellt, sind folgende: An den Muskelfasern angekommen, gehen die durch Verlust der Markscheide zu feinen Axencylindern gewordenen Nervenfasern in verbreiterte und mit eigenthümlich zackigen Umrissen versehene Fasern über, die sich häufig noch mannigfach verzweigen und endlich meistens ganz deutlich abgesetzt enden (s. die Figg 18-20). Die Vergoldung ergibt somit eine völlige Bestätigung der Angaben von $K \ddot{u} h u$ e (mit Ausnahme der Beschreibung der Endknospen), Kölliker, Kra us e und Engelmann.

Was nun die Muskelfasern selbst betrifft, so zeigen sich diese nach Anwendung der Löwit'schen Methode ihrer ganzen Länge, Breite und Dicke nach von deutlich hervortretenden, dunkelroth gefärbten Längsstreifen durchzogen, welche in einer hellen, meist blassröthlich gefärbten, gleichartigen $Z$ wischensubstanz eingelagert sind und in nahezu gleichen Abständen parallel zu einander verlaufen. Diese Längsstreifen bestehen aus hinter einander gelagerten kleinen Körperchen, die bald mehr Kügelchen, bald mehr Strichen ähnlich sind (s. Fig. 18), stellen aber auf verschieden langen Strecken manchmal auch continuirliche Linien dar (Fig. 18 bei a, Fig. 20). Die Länge der Längsstreifen anlangend, so ist diese meist eine sehr verschiedene, manchmal durchziehen sie alle die ganze Länge der Muskelfäden, manchmal sind sie ziemlich kurz (Fig. 20); die Dicke der Streifen ist die von $1 \mu$. im Mittel, beträgt aber häufig auch weniger oder mehr, obwohl sie die. Dicke von $2 \mu$. nicht erreichen. Fin Verhältniss der die erwähnten Streifen zusammensetzenden Körperchen zu den Querstreifen, ähnlich dem an den Muskelfäden der Eidechse beschriebenen, konnte ich nur in ganz einzelnen Fällen wahrnehmen; in diesen (s. Fig. 25) bestanden die Längslinien aus Kügelchen, die in derselben Entfernung, wie die Querstreifen, hinter einander lagen und durch überspringende Linien manchmal deutlich zu den Querstreifen in Beziehung traten. Meistens aber war die Lagerung der erwähnten Körperchen in den Längsstreifen im Verhältniss zu ihrer Lagerung in den benachbarten Streifen eine vollkommen unregel- 
mässige (s. Fig. 18). Statt der beschriebenen Längsstreifen fanden sich in anderen Muskelfäden kürzere Streifen von geraden oder auch wellig gebogenem Verlauf, die in den verschiedensten Winkeln zu einander verliefen und in Folge ihrer hiedurch bedingten unregelmässigen Lagerung den Muskelfäden ein eigenthümlich gesprenkeltes Ansehen verliehen (s. Fig. 23). Auch diese Streifen zeigten eine rosenkranzartige Beschaffenheit, waren aber hie und da auch ungekerbt.

Mit den beschriebenen goldgefärbten Streifen der Muskelfäden stehen die die nervöse Ausbreitung bildenden Fasern in keinem Zusammenhang. Ist der Muskelinhalt stark gefärbt, die Nervenfasern dagegen nur mittelmässig oder gar schwach, so erscheinen zwar manchmal einige der Längsstreifen wie feine direkte Fortsetzungen der Nervenfasern, allein in anderen Fällen, namentlich bei intensiver Schwärzung der Nervenfasern, kann man mit der vollkommensten Sicherheit davon sich überzeugen, dass die Nervenfasern scharf abgesetzt aufhören und die Längsstreifen völlig unabhängig von denselben neben oder unter ihnen verlaufen. Da man nun durch den Wechsel der Einstellung häufig die ganz bestimmte Ueberzeugung gewinnen kann, dass die Nervenfasern über den Längsstreifen der Muskelfasern liegen, ist die scheinbare Fortsetzung der Nervenfasern in die Längsstreifen dadurch leicht zu erklären, dass, indem die Längsstreifen unter den Nervenfasern hinlaufen und eine ähnliche Färbung, wie die letzteren, bei der Goldbehandlung angenommen haben, der Zusammenhang beider nur vorgetäuscht wird. Dass dem so ist, davon überzeugte ich mich auch in einem Falle, in dem ich Anfangs schon einen Uebergang von Nervenfasern in Längsstreifen wahrzunehmen glaubte. Es ging in diesem Falle nämlich eine der zutretenden Nervenfasem in eine diffus gefärbte Masse über, von der ich deutlich Längsstreifen weiter gehen sah (Fig. 18 bei b). Bei genauerer Betrachtung aber ergab sich, dass als Fortsetzung der Nervenfaser nicht die ganze goldgefärbte Masse, sondern nur eine ziemlich unregelmässige Platte (Fig. $18 \mathrm{c}$ ) zu betrachten war, die bei höherer Einstellung erschien als die in die Längsstreifen sich fortsetzende goldgefärbte Masse.

Ich glaube somit eine Verbindung der Nervenfasern mit den nach Anwendung der Löwit'schen Methode in den Muskelfäden auftretenden Längsstreifen, auch für den Frosch in Abrede stellen zu dürfen. Da nun aber die die nervöse Ausbreitung bildenden Fasern in den nach dem $L \ddot{o} w$ it 'schen Verfahren vergoldeten Frosch- 
muskeln deutlich abgesetzteEnden zeigen, und nicht in feine netzförmig zusammenhängende Fasern weiter gehen, andrerseits aber solche netzförmig verbundene Fasern, wie sie Gerlach als Fasern des intravaginalen Nervennetzes beschreibt, in den nach dem Lijwit'schen Verfahren vergoldeten Muskelfäden iiberhaupt nicht aufzufinden waren, ist die Frage aufzuwerfen, ob nicht die mittelst der Löwit'schen Methode dargestellten Iängsstreifen vielleicht dasselbe sind, was Gerlach für intravaginale Nervenfasern hielt, oder ob die Gerlach'schen Nerven nur mit der Methode des genannten Forschers, nicht aber durch das Löwit'sche Verfahren darstellbar sind.

Nach Gerlach treten in den Muskelfasern des Frosches nach Anwendung seiner Methode zweicrlei Gebilde durch ihre Goldfärbung hervor. Die einen sind die intravaginalen Nervenfasern. Diese schildert Gerlach ${ }^{1}$ ) als feine durch Theilung und Wiedervereinigung ein Netz mit, nach den Zeichnungen, langgestreckten Maschen darstellende Fasern, welche durch die Behandlung mit Gold eine dunkelrothe Farbe annehmen und an ihren Rändern zahlreiche, manchmal bis zu völliger Unterbrechung des Zusamimenhangs sich steigernde Einkerbungen zeigen, wodurch sie ein den variküsen Fasern des Cercbrospinalorgans ähnliches, rosenkranzartiges Ansehen eriangen. 'Trotz ihrer zahlreichen 'Theilungen und Wiedervereinigungen behalten die intravaginalen Nervenfasern einen constanten Durchmesser von 0,001-0,015 Mm. Die zweite Art der goldgefärbten Elemente in den Muskelfäden sind die sogenanaten Sprenkelungen Gerlach's. Diese Gebilde besitzen nach G erlac $\mathrm{h}^{2}$ ) eine kaum messbare Breite, welche jedenfalls unter $0,001 \mathrm{Mm}$. liegt, sind etwas länger als breit und machen hie und da selbst einen faserähnlichen Eindruck. Nach der Fig. 10 von Gerla ch, welche ein Präparat darstellt, an wclchem ein Theil der quergestreiften Substanz aus dem Sarcolemm herausgedrängt war, und nach der Aehnlichkeit der an Präparaten nach der Löw it'schen Methode gesehenen Bilder, wobei auch der contraktile Inhalt aus dem Sarcolemm vorgequollen war, muss ich die Längsstreifen der Gold-Ameisensäure-Präparate von Muskelfäden für Analoga der faserähnlich gewordenen Sprenkelungen Gerlachs erklären. Auf der anderen Seite aber haben die Längsstreifen die

1) l. c. p. 48 .

2) 1. c. p. 50 . 
den Sprenkelungen Ge rl a ch s abgehende rosenkranzartige Beschaffenheit und nach den mitgetheilten I ickenverhältnissen Dimensionen, die mit den Angaben Gerlach's uber die Dicke der intravaginalen Nervenfasern ziemlich äbereinstimmen.

Hierzu kommt noch, dass den erwähnten Längsstreifen vollkommen gleiche Gebilde manchmal in den nach dem Löwit'schen Verfahren vergoldeten Muskelfäden, unregelmässig hin und her gebogen verlaufen, wobei durch Deckung der wellig gebogenen Gebilde, der Anschein eines netzförmigen Zusammenhanges derselben hervorgerufen werden kann (Fig. 22). Indem aber nach dem blossen Vergleich der mittelst der Lö̈wit'schen Methode gewonnenen Bilder mit den Angaben und Abbildungen Gerlachs nicht zu urtheilen war, wandte ich die Gerlach'sche Methode genau in der von ihm beschriebenen Weise an, fand aber auch an den auf diese Weise erhaltenen Präparaten stets nư den Längstreifen der Gold-Ameisensüure-Präparaten vollkommen analoge, rosenkranzartige Streifen, deren Darstellung nur nicht, wie bei Anwendung der Löwit'schen Methode, in der ganzen Länge und Breite der Muskelfälen gelungen war. An einigen der nach der Methode Gerlachs vergoldeten Muskelfasern aber, die ich Muskelstiickchen des Oberschenkels und Oberarms eines vor 9 Stunden getödteten Frosches entnommen hatte, fand ich ganz dieselbe Nervenausbreitung, wie sie Kühne zuerst beschrieben und wie sie nach Anwendung des Löwit'schen Verfahrens auftritt; ich habe eine solche mit der Methode Gerlachs dargestellte Nervenendigung in Fig. 21 abgebildet, aus welcher zu ersehen ist, dass die Muskelfasern, obwohl die Nervenfasern deutlich gefärbt sind, farblos sich darstellen und intravaginaler Streifen vollkommen entbehren, dass ferner die Fasern der nervösen Ausbreitung, auch nach Anwendung der Gerlach'schen Methode, nicht in feinere Fasern sich fortsetzen, sondern in Uebereinstimmung mit den mittelst des Löwit'schen Verfahrens erhaltenen Befunden, in der That frei enden, wie $K \ddot{u} h n e$ dies beschrieben und nach ihm viele andere Autoren es bestätigt haben. Es folgt nun hieraus, dass in Bezug auf das durch Vergoldung zur Anschauung kommende Verhalten der Nerven- und Muskelfasern des Frosches eine vollkommene Uebereinstimmung besteht, zwischen dem Erfolg der Löwit'schen Methode und den Resultaten, wie sie sich mir aus der Anwendung des Gerlach'schen Verfahrens ergaben, dass somit der Einwurf mir nicht mehr gemacht werden kann, es könnten die intra- 
vaginalen Nervenfasern sich vielleicht gegen das Löwit'sche Verfahren völlig indifferent verhalten und seien nur mittelst der Gerlach'schen Methode, durch diese aber mit Sicherheit, darzustellen. In Anbetracht der vollkommen gleichen Resultate des Löwit'schen, Gerlach'schen und (nach Fig. 20) auch des Cohnheim'schen Verfahrens nun muss ich mit aller Bestimmtheit die Sätze aufstellen: 1) Die die motorische Nervenendigung an den Muskelfäden des Frosches bildenden Fasern enden frei. 2) Das intravaginale Nervenfasernetz von Gerlach existirt nicht.

Für den sub 2 aufgestellten Satz und für die freie Endigung der Nervenfasern gebe ich noch folgende Beweise: Durch Anwendung der Löwit'schen und Gerlach'schen Methode gelingt es, die nervöse Ausbreitung vollkommen gefärbt $\mathrm{zu}$ erhalten, während die Muskelfäden selbst nahezu farblos bleiben, d. h. die erwähnten Streifen in denselben nicht zur Darstellung gelangen. Ls ergibt sich hieraus, dass ein Unterschied der durch Gold darstellbaren Streifen der contraktilen Substanz und der die nervöse Ausbreitung bildenden Fasern existirt in Bezug auf ihr Verhalten gregen Gold. Ein solcher Unterschied aber wäre, falls die Streifen auch von nervöser Natur waren, nicht leicht zu erklären. - An Muskelfäden, die nach der Löwit'schen Methode vergoldet wurden, ist man, namentlich nach Maceration der vergoldeten Muskelstückchen in Wasser, im Stande, durch Quetschen den contraktilen Inhalt aus dem Sarcolemm zu pressen. In solchen Präparaten nun findet man Bilder, wie das in Fig. 19 dargestellte, wo die die nervöse Ausbreitung bildenden Fasern in vollkommen gleicher Lage, wie sie an den mit gefärbten Längsstreifen versehenen Muskelfäden sich befinden, wie isolirt auf einem mehr weniger farblosen Grunde liegen. Es kann dies wohl nicht anders gedeutet werden, als so, dass in diesen Fällen die nervöse Ausbreitung, auf oder unter dem bei der Vergoldung nahezu farblos bleibenden Sarkolemm liegend, von dem durch den Druck hinausgepressten mit goldgefärbten Längsstreifen versehenen Inhalt der Muskelfasern sich getrennt hat. Mit dieser Erklärung will ich jedoch die auch noch mögliche Deutung dieser Erscheinung, dass nämlich die Nervenfaserausbreitung durch den Druck in diesen Fällen ganz und gar von den Muskelfäden abgelöst worden sei, nicht völlig ausschliessen, doch scheint mir die ersterwähnte Annahme immer noch mehr Wahrscheinlichkeit für 
sich zu haben, da man nach dem erwähnten Verfahren an den Präparaten erstens zusammengefaltete, homogene Membranen sieht, die man wohl nur für leere Sarcolemmschläuche halten kann, und da zweitens solche Membranen unter oder über den isolirten Nervenendigungen, wenngleich nicht in allen Fällen, wahrzunehmen sind. Die Trennung der Nervenendigung von dem gefärbten Muskelinhalt und ihr vollkommenes Intaktbleiben hierbei scheint mir aber jedenfalls zu beweisen, dass eine Fortsetzung der Nervenfasern in, die Muskelfäden ihrer ganzen Länge nach durchziehende, feine Fasern nicht besteht, da sonst wohl in einigen Fällen Theile von intravaginalen Fasern an den Nervenfasern hängen geblieben oder die nervöse Ausbreitung zerrissen, jedenfalls aber nicht in so vollkommener Integ rität erhalten worden wäre. -

Dass nun Ger la ch ein intravaginales Nervennetz angenommen, beruht darauf, dass erstens Bestandtheile der contraktilen Substanz in hervorstechender Weise mit Gold sich färben und hierbei Fasern ähnlich werden, dass dieselben zweitens ein den rosenkranzförmigen feinen Nervenfasern ähnliches Ansehen zeigen und manchmal auch netzförmig unter einander zusammenzuhängen scheinen und dass drittens, weil die goldgefärbten Streifen der contraktilen Substanz unter den Nervenfasern weglaufen, bei der ähnlichen Farbe beider eine Continnität zwischen ihnen leicht vorgetäuscht werden kann.

Die faserartigen Gebilde von rosenkranzartiger Beschaffenheit, die Gerlach für Nervenfasern gehalten, sind die Längsstreifen der Gold-Ameisensäure-Präparate, deren scheinbar netzförmiger $\mathbf{Z u -}$ sammenhang aus Fig. 22 sich ergibt. Dass die letzteren wirklich Gerlach's intravaginalen Nervenfasern entsprechen, ergibt sich aus der gleichen Färbung, dem gleichen gekerbten Aussehen und den nahezu ähnlichen Dickenverhältnissen derselben und der intravaginalen Nervenfasern Gerlach's.

Indem somit die intravaginalen Nervenfasern Gerlach's als Theile der Muskelsubstanz aufgefasst werden müssen, andererseits aber die Sprenkelungen der Muskelfäden, auch nach den Angaben Gerlach's ebenfalls Bestandtheile der contraktilen Substanz sind, handelt es sich noch um das Verhältniss dieser beiden Gebilde zu einander. Nach Anwendung der Löwit'schen Methode treten in den Muskelfäden des Frosches, wie ich oben erwähnt, zweierlei goldgefärbte Elemente hervor. Dieselben sind entweder Längsstreifen von rosenkranzartiger Beschaffenheit, die, von verschiedener 
Länge, untereinander und mit der Längsrichtung der Muskelfäden parallel verlaufen, oder kurze, mehr strichähnliche Streifen von unregelmässigem Verlaufe, welche den Muskelfäden ein gesprenkeltes Ansehen verleihen. Da sich nun der Nachweis liefern lässt, dass die kurzen Streifen dasselbe sind, wie die parallelen Längsstreifen, weil sie im Verlaufe einer Muskelfascr sich deutlich zu parallelen Längsstreifen an einander lagern können (s. Fig. 24), so sind die beiden nach Anwendung der Löwit'schen Methode hervortretenden und theils durch ihre Länge, theils durch ihren Verlauf sich unterscheidenden goldgefärbten Streifen einerlei Art. Wie erwähnt, bestehen die goldgefärbten Längsstreifen und die kurzen Streifen aus kleinen, theils Kügelchen, theils Strichen ähnlichen Gebilden, die sich linienförmig an einander reihen. Ist die lineare Anordnung dieser kleinen Körperchen nun vollkommen ausgeprägt, so entstehen parallele Längsstreifen, reihen sich dagegen nur wenige Körperchen an einander und erhalten die dadurch entstehenden Streifen, viclleicht in Folge der präparativen Behandlung, eine nicht parallele, sondern unregelmässige Lagerung, so entstehen die Sprenkelungen der Muskelfäden an Gold-Ameisensäure-Präparaten. Die Sprenkelungen Gerlach's nun entsprechen zu einem Theil diesen Sprenkelungen, zu einem anderen Theil (in den Fällen nämlich, in welchen nach Gerlach nur ein punktirtes Ansehen der Muskelfälen vorhanden war) sind sie vielleicht den noch mehr isolirten punktförmigen Körperchen gleich, deren lineare Anordnung sich nicht ausgeprägt hatte.

Die von Gerlach als faserähnlich bezeichneten Sprenkelungen ferner sind gleich Längsstreifen, die durch das Gerlach'sche Verfahren nur in geringer Länge dargestellt worden waren.

Dass nun in der That dieselben Formelemente der Muskelfasern von Gerlach theils als Nervenfasern, theils als Bestandtheile der contraktilen Substanz gedeutet worden sind, dafür spricht auch der Umstand, dass die intravaginalen Nervenfasern und die Sprenkelungen der Muskelfasern nach den Angaben Gerlachs stets dieselbe Farbe nach der Goldbehandlung zeigen. $\mathrm{Zu}$ erklären habe ich noch, warum Gerlach in einer Muskelfaser neben den intravaginalen Nervennetzen auch die Sprenkelungen auffinden konnte, und ferner, auf welche Weise Gerlach zur Annahme eines Zusammenhangs zwischen den als intravaginale Nervenfasern und den als Sprenkelungen der Muskelfäden aufgefassten Gebilden gelangt 
ist. Den ersterwähnten Befund Gerlach's, der durch dessen Fig. 11 versinnlicht ist, bin ich nur auf die Weise zu erklären im Stande, dass die erwähnten kleinen Körperchen, die zur Bildung von Längsstreifen zusammentreten, theils zu langen und unregelmässig verlaufenden Streifen sich vereinigt hatten, theils völlig isolirt geblieben oder nur zu ganz kurzen Streifen zusammengetreten waren; die Möglichkeit dieser Erklärung ergibt sich wenigstens daraus, dass in einzelnen Muskelfäden, die nach der Löwit'schen Methode vergoldet worden waren, an ein und derselben Stelle theils parallele Längsstreifen theils Sprenkelungen zu sehen waren. Auf welche Weise Gerlach zu der Annahme eines Zusammenhangs zwischen intravaginalen Nervenfasern und den Sprenkelungen der Muskelfasern kam, war ich aus meinen Befunden nicht zu ermitteln im Stande.

Was noch die letzte Klasse der Wirbelthiere, die Fische, anbelangt, so gelang es mir bei diesen, trotz wiederholter Versuche, nicht, das terminale Verhältniss der Nervenfasern an den Muskelfäden aufzudecken. Ich verwandte zur Untersuchung hauptsächlich die Augenmuskeln vom Hecht und rom Karpfen, später auch die Flossenmuskeln dieser Thiere und verfuhr hierbei ganz nach der Methode, die ich oben (im Absatz über die Nervenendigung bei den Vögeln) beschrieben habe. Gefunden habe ich bei diesen Versuchen, dass die zwischen den Muskelfasern hinlaufenden Nervenfasern, wie es ja nach den Untersuchungen von Joh. Müller und E. Brü cke ${ }^{1}$ ) längst bekannt ist, vielfachen Theilungen unterliegen, dass sie in Folge dieser sehr fein und endlich durch Aufhören des Goldnieder. schlags unsichtbar werden. Ein Uebergang in feine, nach dem Innern der Muskelfäden ausstrahlende Fasern konnte nie nachgewiesen werden, dagegen fanden sich an einigen Stellen der Muskelfasern durch Gold gefärbte, spindelförmige Gebilde von der Farbe der goldgefärbten, markhaltigen Nervenfasern, die auf der einen Seite zugerundet oder zugespitzt endigten, auf der andern aber in feine, endlich auch verschwindende Fortsätze übergingen. Einen Zusammenhang dieser Fortsätze mit den feinen Fortsetzungen der Nervenfasern konnte ich nie nachweisen, wonach ich nicht im Stande bin, die er-

1) Handb. d. Physiologie v. Joh. Mülter. 4. Aufl., Bd. I, pag. 524. 
wähnten spindelförmigen Gebilde als nervös und als Endigungen der Nervenfasern aufzufassen.

Ich habe jetzt noch einen Ueberblick zu geben über die Gesammtverhältnisse der Endigung der motorischen Nerven bei den vier ersten Klassen der Wirbelthiere. Zunächst ist hierbei zu berühren, in welcher Weise die durch Anwendung der Löwit'schen Methode bei den drei ersten Wirbelthierklassen gewonnenen Befunde $\mathrm{zu}$ den Angaben Gerlach's sich verhalten. Meine Darstellung sowie die beigegebenen Abbildungen zeigen, dass einmal das Vorhandensein von Endplatten Gerlach gegenüber jedenfalls mit aller Entschiedenheit festzuhalten ist. Da nun aber weder bei den Säugern, noch bei den Vögeln, noch bei den Reptilien Fortsetzungen der die Endplatten bildenden Fasern jemals nachzuweisen waren, sondern diese Fasern stets scharf umrissen enden, muss, wie für die Amphibien, so auch für die drei ersten Klassen der Wirbelthiere die terminale Bedeutung der Endplatten behauptet und die Existenz intravaginaler Nervennetze in Abrede gestellt werden. $\mathrm{Zu}$ bemerken habe ich ferner, dass die an den Muskelfasern bei den drei ersten Wirbelthierklassen erhaltenen Befunde die oben (im Absatze über die Nervenendigung beim Frosche) aufgestellte Behauptung bestätigen, wonach die goldgefärbten, durch ihre rosenkranzartige Beschaffenheit ausgezeichneten Streifen als Bestandtheile der contraktilen Substanz zu betrachten sind. Es zeigen nämlich auch die Muskelfasern der Säugethiere (hauptsächlich in Fällen, in denen mir die Darstellung von Endplatten nicht gelungen war oder in welchen dieselben nur schwach gefärbt hervortraten) deutliche Längtstreifen, die denen der Vögel, der Reptilien und Amphibien sehr ähnlich sind, zuweilen ungekerbte Linien darstellen, zuweilen, wie in den Muskelfasern der Eidechse, aus hintereinander liegenden kleinen Körperchen bestehen, welche durch Aneinanderreihung in der Querrichtung Querstreifen bilden (s. Fig. 26). Schon dieses Verhältniss zwischen Längs- und Querstreifen beweist, dass diese Formelemente Theile der Muskelsubstanz sind; es wird aber diese Annahme auch dadurch noch bestätigt, dass bei allen Klassen der Wirbelthiere die Längsstreifen ein anderes Verhalten gegen Gold zeigen, als die Fasern der nervösen Ausbreitung. Auf die bestimmte Entscheidung der Frage aber, welche Theile der contraktilen Substanz es sind, welche mit 
Gold in so hervorstechender Weise sich färben, darauf konnte ich bis jetzt theils wegen Mangel an Zeit, theils, weil ich die vorliegende Mittheilung nur als eine kurze Bestätigung der Existenz von Endplatten betrachtete, nicht eingehen. Demnach lasse ich es unentschieden, ob die Längsstreifen der Muskelfäden bei allen untersuchten Thierklassen denselben Bestandtheilen der contraktilen Substanz entsprechen und in welcher Weise die sie zusammensetzenden Körperchen $z u$ den anisotropen und isotropen Elementen und $z u$ den interstitiellen Köruchenreihen sich verhalten ${ }^{1}$ ).

Vergleichen wir nun die durch das Verfahren von Löwit dargestellten Formen der motorischen Nervenendigung bei den vier ersten Klassen der Wirbelthiere unter einander, so ergibt sich, dass bei den drei ersten Klassen die in Eorm von Endplatten auftretende Nervenendigung im Wesentlichen aus verbreiterten Fasern besteht, die, auf einen verhältnissmässig sehr kleinen Raum der Oberfläche der Muskelfasern sich begrenzend, hier sich gegeneinander biegen, während bei den Amphibien die nervöse Ausbreitung über eine ziemlich grosse Fläche der Muskelfäden sich erstreckt und die Terminalfasern nur parallel neben einander hinlaufen ${ }^{2}$ ). Solche aus parallelen Fasern bestehende Endausbreitungen kommen aber nicht bei den Amphibien allein, sondern, wie schon erwähnt, auch schon bei den Vögeln, aber selten, vor. Aus dem Zusammenhalt der motorischen Nervenendigung bei den Säugethieren, Vögeln, Reptilien und der Nervenendigung bei den Amphibien ergibt sich nun, dass ein Unterschied zwischen denselben der Hauptsache nach nicht besteht, da als wesentlicher Charakter der Nervenendigung in übereinstimmender Weise verbreiterte Fasern sich darstellen, die nur durch die Verschiedenheit ihrer Lagerung, Länge und ihres Verhältnisses zu der Grösse des bedeckten Theils der Muskelfasern bei den verschiedenen der genannten Wirbelthierklassen verschiedene Endausbreitungen bilden. Analysiren wir aber die Verhältnisse der motorischen Nervenendigung bei den erwähnten Klassen der Wirbelthiere noch genauer,

1) Was noch die Lage der Nerveuausbreitung an den Muskelfäden anbelangt, so ist es mir nicht gelungen, definitive Aufschlüsse darüber zu erlangen, ob dieselbe ausser- oder innerhalb des Sarcolemms gelegen ist.

2) In einzelnen Fällen finden sich auch bei Amphibien, wie ich jüngst bei Salamandra maculata gefunden, deutliche Endplatten, denen der Vögel sehr ähnlich. 
so ergibt sich erstens, dass das charakteristische Moment der Nervenendigung ganz allein in den das Ende des Axencylinders darstellenden, verbreiterten Fasern liegt, da weder die hügelartigen Erhebungen (die manchmal schon beim Menschen, dann auch bei den Vögeln und stets bei den Amphibien fehlen) noch die (den Amphibien stets mangelnde) granulirte Plattensohle als wesentliche Merkmale angesehen werden können. Als zweites Ergebniss der vergleichenden Betrachtung der Nervenendigung aber stellt sich, wie mir scheint, ein Fortschritt dar in Bezug auf die Erkenntniss des allgerneinen Princips der Nervenendigung im quergestreiften Muskel. Schon Kühne macht in Strickers Handbuch der Gewebelehre pag. 163-165 den Versuch, die Endigung der Muskelnerven unter einem gemeinsamen Gesichtspunkt aufzufassen und koinmt hierbei zu dem Schlusssatze: "An allen quergestreiften Muskeln entspricht das Ende des Axencylinders einer Ausbreitung mit bedeutend vermehrter Oberfläche, welche stets durch eine flach ausgebreitete Verzweigung gebildet wird. Diese Nervenendplatte ist bald mehr membranartig, bald einem Fasersysteme vergleichbar". Ist'nun auch die K ühne'sche Auffassung, nach dem durch die Vergoldung ebenfalls bestätigten Princip der Oberflächenvermehrung durch Verzweigung, vollkommen richtig, so scheint sie mir doch das Grundprincip der motorischen Nervenendigung noch nicht einzuschliessen. Gehe ich, bei dem Versuche, dieses darzulegen, von der Nervenendigung bei den Säugethieren aus, so ist die plattenförmige Endausbreitung bei diesen dadurch gebildet, dass das verbreiterte Nervenfaserende sich vielfach verzweigt und die einzelnen Terminalfasern gegen einander sich hinkrümmen. Eine ziemlich einfache Nervenendigung nun zeigt Fig. 3 (vom Menschen); dieser liegt schon weniger eine Oberfächen-Vermehrung, durch Verzweigung zu Grunde, sondern das Ende des Axencylinders wird nur von verbreiterten und in Anschwellungen endenden Fasern dargestellt, die während ihres Verlaufs sich allerdings, aber viel weniger, als in den übrigen Endplatten, verzweigen und, indem sie nicht auf einem kleinen Raume sich gegeneinander biegen, keine ausgeprägte Endplatte bilden. Dass im Wesentlichen die Nervenendigung an der quergestreiften Muskelfaser nur von einer Verbreiterung oder Anschwellung des Axencylinders gebildet wird, scheint sich mir nocb mehr aus der Fig. 9 A zu ergeben, nach welcher die zutretende Nervenfaser einfach in zwei verbreiterten Anschwellungen endet. Aehnliche Fälle, 
in welchen die durch Verzweigung des Nervenfaserendes ausgesprochene Bildung eigentlicher Endplatten zurücktritt und in denen mehr einfache Anschwellungen als die Nervenendigung charakterisirend hervortreten, zeigen die Figg. $9 \mathrm{~B}$ u. $3 \mathrm{~A}-\mathrm{C}$. Aus dem Zusammenhalt der Figg. $3,3 \mathrm{~A}-\mathrm{C}$ u. $9 \mathrm{~A}$ u. $\mathrm{B}$ mit den übrigen Formen von Endplatten ergibt sich aber, wie mir scheint, dass die Endplatten nur eine auf Verzweigung beruhende Complication der Endigung in einfachen Anschwellungen des Axencylinders darstellen. Unter diesem Gesichtspunkt lässt sich auch die Nervenendigung bei den Amphibien auffassen, nur erlangt bei diesen die terminale Anschwellung des Axencylinders stets die Peschaffenheit langer Fasern und complicirt sich auch meistens durch Verzweigung. Die erwähnte Annahme, wonach der motorischen Nervenendigung im Wesentlichen eine Anschwellung der Nervenfasern zu Grunde liegt, erhält noch mehr Wahrscheinlichkeit durch einige bekannte Thatsachen bei Wirbellosen, namentlich durch das bekannte Verhältniss der Nervenendigung an den Muskelfasern von Milnesium tardigradum, welche von Do y er è ${ }^{1}$ ) entdeckt und später von Greeff ${ }^{2}$ ) bestätigt wurde. An diese Thatsache reihen sich noch die Befunde von Quatrefage s ${ }^{3}$ ) bei Eolidina und Amphioxus, von Kölliker ${ }^{4}$ ) bei einer Chironomuslarve, von Meiss $n e r^{5}$ ) bei Mermis und Ascaris, von Wed $l^{6}$ ), Walt er ${ }^{7}$ ) und $\mathrm{Munk}^{8}$ ) bei anderen Nematoden, bei denen die Endigung der Nerven in Anschwellungen auch von Bütschli ${ }^{9}$ ) und Krause ${ }^{10}$ ) später noch bestätigt wurde. Nach allen diesen Befunden ist es klar, dass bei Wirbellosen häufig die Nervenfaser mit einer an die Muskelfaser sich anlegenden, einfachen Anschwellung endet, eine That-

1) Memoire sur les tardigrades. Ann. des sciences natur. 2. Serie. 1840.

Pl. 17. Fig. 1-4.

2) Archiv f. mikr. Anatomie v. M. Schultze. Bd. I. pag. 101.

3) Ann. d. scienc. nat. II. Série 1843. Taf, XIX. pag. 299.

4) Mikr. Anat. Bd. II. I. Hälfte. pag. 238.

5) Zeitschr. f. wiss. Zool. Bd. V. 1854. pag. 234 und Bd. VII. 1856. pag. 26.

6) Wien. Sitzungsber. Bd. VIII. pag. 298.

7) Zeitschr. f. wiss. Zool. Bd. VII(. pag. 163.

8) Götting. Nachr. 1858. Nro. 1. pag. 11.

9) Arch. f. mikr. Anat. v. M. Schultze. Bd. 10. pag. 79.

10) Nach einer Notiz im Lehrb. d. Anatomie v. C. F. Th. Krause, Hannov. 1876. pag. 499. 
sache, die im Zusammenhalt mit den durch das L ö wit'sche Verfahren bei den höchsten Klassen der Wirbelthiere erhaltenen Resultaten vielleicht zu dem Satze berechtigt: Dem Prinzip uach kommt die Endigungder Nervenfaser an derwillkürlichen Muskelfaserdadurch zu Stande, dassdieNervenfaser durch eine terminale Anschwellung mit der contraktilen Substanz in Berührung tritt ${ }^{1}$ ). Diese terminale Anschwellung wird bei den höheren Klassen der Wirbelthiere meist faserartig und complicirt sich durch Verzweigung, wodurch theils terminale Fasersysteme (bei den Amphibien) theils Endplatten (bei den Reptilien, Vögeln und Säugern) gebildet werden. -

Dem genannten Principe nun scheint der von Gerlach ${ }^{2}$ ) angezogene Befund Kleinenbergs ${ }^{3}$ ) an Hydra einigermassen zu widersprechen. Nach dem letztgenannten Autor sind die zwischen Ektoderm und Entoderm verlaufenden Muskelfasern dieses Thieres Fortsätze von Ektodermzellen, d. h. sie stehen in Continuität mit den Reiz empfindenden und leitenden Zellen. Nimmt man aber an, dass mit der Vereinfachung der Organisation die Contiguität zwischen Reiz leitenden und sich verkürzenden Fasern in eine Continuität über geht, was allerdings hypothetisch ist, so lässt sich auch das Verhältniss bei Hydra dem erwähnten Prinzipe unterordnen und muss mit diesem sogar in ähnlicher Weise in Beziehung gebracht werden, nachdem für die Muskelfaser der höheren Wirbelthiere die Existenz

1) Nach dem übereinstimmenden Ergebniss sämmtlicher mittelst der Goldmethode angestellter Untersuchungen ist es klar, dass bei den höheren Wirbelthieren zwischen Nerven- und Muskelsubstanz stets nur eine Contig. nität, nie eine Continuität vorhanden ist Der Contakt zwischen contraktiler und nervöser Substanz scheint aber ein direkter nur bei den Amphibien zu sein, weil diesen dis granulirte Plattensohle fehlt, bei den übrigen Klassen der Wirbelthiere scheint dasselbe durch die feinkörnige Protoplasmaunterlage vermittelt zu werden, die von den Autoren für die Endplatten beschrieben ist. Doch lässt sich an Goldpräparaten, so viel ich gesehen babe, niemals eine Beziehung der Endplatten-Fasern zu dem vielleicht als gefärbte Plattensohle zu betrachtenden Goldniederschlag, den ich bei den Endplatten beschrieben habe, nachweisen.

2) l. c. pag. 15 und 56 .

3) Hydra. Eine anatomisch-entwicklungsgeschichtliche Untersuchung. Leipz. 1872. pag. 11. 
intravaginaler Nervenfasernetze, mit welchen Gerlach die Neuromuskelzellen von Hydra verglichen, nach dem Voranstehenden bestimmt in Abrede zu stellen ist.

Anlässlich einer Angabe von W. Kra use ${ }^{1}$ ), dass auch an den Muskelfäden des Herzfleisches die Nervenfasern mit Endplatten endigen, habe ich auch den Herzmuskel (des Hundes) unter Anwendung der Löwit'schen Methode vergoldet. Die hiebei erhalteneen Resultate sind folgende: Unter den Muskelfasern, welche bei der Vergoldung an vielen Stellen der Muskelstïckchen vollkommen farblos geblieben waren, verlaufen in ungemein grosser Menge und meist parallel der Längsrichtung der Muskelfällen gestellt, intensiv geschwärzte Nervenfasern von sehr verschielener Dicke, deren hervorstechendstes Merkmal darin liegt, dass sie unter einander sehr zahlreiche Verbindungen eingehen, wodurch langmaschige Netze zu Stande kommen. Diese den Muskelfäden parallelen Nervenfasern kommen durch allmähliche Theilung aus sehr dicken Fasern hervor (s. Fig. 31), die häufig senkrecht oder schief, manchmal auch parallel zur Längsrichtung der Muskelzüge herantreten. Was ihre Endigung anbelangt, so waren Endplatten niemals aufzufinden, statt derselben sah ich, ausser scheinbar frei endenden Fasern, auf die ich gleich zurückkommen werde, nur netzförmige Vereinigungen der Nervenfasern, welche sowohl unter den feinen, wie unter den gröberen Fasern zu Stande kommen (s. d. Figg. 28 und 29). Nun ist es nicht unwahrscheinlich, dass diesen Nervenfasernetzen die Bedeutung von Endnetzen zukommt; es ist nämlich die netzförmige Verbindung der Nervenfasern ungemein häufig zu constatiren, ferner verbinden sicb auch die feinsten Fasern auf diese Weise mit einander und endlich ist nie eine andere Art der Endigung mit, Sicherheit nachzuweisen. Gegen die erwähnten freien Enden der Nervenfasern nämlich ist einzuwenden, erstens, dass in diesen Fällen die betreffenden Nervenfasern nicht weiter zur Darstellung gelangt sein können und zweitens dass an diesen Enden (wie Fig. 28 bei a eines zeigt) kein besonderes die Endigung kennzeichnendes Verhältniss aufzufinden ist, sondern die Fasern wie abgerissen aufhören. Eine andere Erscheinung, die mit

1) Anatomie d. Kaninchens. Leipz. 1868. pag. 178. 
der Endigung in Beziehung gebracht werden könnte, ist eine hie und da sich findende lokale Goldfärbung der Muskelfäden; an manchen Stellen der letzteren nämlich findet sich ein eigenthümlicher körniger Goldniederschlag, der eine mehr oder minder lange Strecke der Muskelfäden einnimmt, an manchen Punkten an intensivsten vorhanden ist, und von hier aus nach beiden Seiten gegen die Enden der Muskelfäden hin allmählich verschwindet (s. Fig. 32). In diesem Niederschlag finden sich nicht selten dieselben linieuförmigen Punktreihen, theils Quer- theils Längsstreifen darstellend, welche ich von den vergoldeten willkürlichen Muskelfasern beschrieben habe. Dass sich nun mit den erwähnten goldgefärbten Stellen der Muskelfasern die Nervenfasern verbunden hätten, war ich nie mit Sicherheit wahrzunehmen im Stande, doch will ich die Möglichkeit eines sulchen Zusammenhangs nicht gänzlich in Abrede stellen.

Was nun die Lagerung der beschriebenen, wahrscheinlich als terminal zu betrachtenden Nervenfasernetze im Verhältniss zu den Muskelfäden anhelangt, so zeigt vor Allem das Querschnittsbild (Fig. 30), dass die Nervenfasern (wenigstens die stärkeren Calibers mit Bestimmtheit) zw ischen den Muskelfäden gelegen sind. Die feinsten Fasern konnte ich an Querschnitten allerdings nicht mit Sicherheit auffinden, doch fanden sich weder am Querschnitt noch auch am Längsschnitt Andeutungen vor, dass die Nervenfasern in das Innere der Muskelfäden eintreten. Ich glaube somit auch für die Nerven des Herzens einen intravaginalen oder intramuskulären Verlauf in Abrede stellen zu dürfen. - Die Beschaffenheit der Nervenfasern im Herzen anlangend, so zeigen dieselben erstens eine sehr wechselnde Dicke. Die feinsten Fasern, die ich wahrnahm, massen 0,001 Mm., die mittleren 0,003-0,010 Mm., die dicksten 0,015 bis 0,027 Mm. im Mittel. Ausgezeichnet sind die vergoldeten Nervenfasern im Herzen zweitens durch Verdünnungen uud Verdickungen, die sich während des Verlaufs in sehr ausgesprochener Weise und sehr zahlreich auftretend vorfinden (s. die Figg. 27-29) und dann durch intensiv geschwärzte Stellen, die theils längere und gleichmässig dicke Strecken der Fasern betreffen (s. Fig. 28 bei b), theils auf die verdickten Stellen sich beschränken (Fig. $28 \mathrm{u} .29 \mathrm{c}$ ), welche dadurch als in eigenthümlicher Weise hervortretende intercalare Anschwellungen sich darstellen. Diese intensive Färbung, welche manche Stellen der Nervenfasern bei der Vergoldung annehmen, ist aber nicht, wie man vermuthen könnte, dadurch bedingt, dass diese 
Stellen von markhaltigen Strecken der Nervenfasern gebildet werden. Es zeigt, nämlich die Behandlung des lebenswarmen Herzmuskels mit Osmiumsäure, dass zwischen den Huskelfäden keine entsprechenden tintenschwarz gefärbten Stellen hervortreten, sondern die Schnitte des Muskels stellen sich ihrer ganzen Ausdehnung nach an jeder Stelle gleichmässig gelblich oder grünlich gefürbt dar.

Fasse ich nun das Verhalten der Nerven in der Herzmuskulatur noch kurz zusammen, so ergibt sich, dass die durch Theilung aus den dicksten Nervenfasern hervortretenden Fasern zwischen den Muskelfäden und parallel deren Lüngsaxe hinziehen und hiebei durch Verbindung unter einander Netze mit sehr langgestreckten Maschen bilden. Während ihres Verlaufs verdünnen und verdicken sich die Fasern sehr häufig und zeigen oft eigenthümliche durch besonders intensive Goldfärbung ausgezeichnete Stellen. -

$\mathrm{Zu}$ bemerkew habe ich noch, dass, wenn die terminale Bedeutung der beschriebenen intermuskulären Nervennetze sich bestätigt, in Bezug auf die Nervenendigung im Herzen eine sehr grosse Analogie mit den von Löwit ${ }^{1}$ ) in jüngster Zeit über die Nerven der glatten Muskulatur gemachten Angaben sich herausstellen würde.

Die vorliegenden Untersuchungen sind im hiesigen histologischen Laboratorium von Herrn Prof. Kollmann ausgeführt worden. Für die mir zu'Theil gewordene überaus freundliche Unterstützung spreche ich Herru Prof. K oll mann meinen besten Dank aus.

München, im April 1876.

Kurz vor Abschluss vorliegender Arbeit erhielt ich eine jüngst erschienene Abhandlung von Dr. Le o Gerlach "Ueber die Nervenendigungen in der Muskulatur des Froschherzens«. In dieser Abhandlung stimmt $L$. Gerlach in Bezug auf die Nervenendigung in der willkürlichen Muskelfaser ganz mit J. Gerlach überein, in Bezug auf die Nervenendigung im Herzen des Frosches hat er Nervennetze gefunden, die theils die Muskelbündel, theils die Muskelzellen umspinnen. Von den letzteren Nervennetzen, die L. Gerlach intramuskuläre nennt, sollen feine Nervenfibrilien auch in das Innere der Muskelzellen eindringen können. Was nun die Aufrechterhaltung

1) l. c. 
der Angaben von J. Gerlach durch L. Gerlach anbelangt, so muss ich auf die Resultate, die ich der Methode von Löwit verdanke, gestützt, auch diesem Autor gegenüber die Existenz der intravaginalen Nervennetze in Abrede stellen, da er keine neuen Beweisgründe für das Vorhandensein derselben beibringt. In Betreff der Befunde L. Gerlach's über die Nerven des Froschherzens steht mir kein Urtheil zu, da ich über die letzteren keine Untersuchungen gemacht habe. Die Angaben des genannten Autors stimmen übrigens, bis auf die Annahme, dass feine Nervenfasern ins Innere der Muskelfäden eindringen können, ziemlich vollständig mit den Resultaten überein, die ich in Bezug auf das Verhalten der Nerven im Herzen des Hundes erhalten. Bei dem letzteren aber konnte ich, wie erwähnt, nie ein Eindringen von Nervenfasern in Muskelfäden constatiren.

\section{Erklärung der Abbildungen auf Tafel XXV und XXVI.}

(Sämmtliche Figuren mit Ausnahme von Fig. 20 und 21 sind nach GoldAmeisensäure-Präparaten gezeichnet.)

Fig. 1 u. 2. Muskelfäden mit, Endplatten in Flächenansicht vom Menschen.

1 mit Seibert Immers. VI/I. 2. m. VII/O.

a Endanschwellungen der Terminalfasern.

b Zugerundetes Ende derselben.

c Zugespitztes Ende derselben.

d In den Verlauf der Fasern eingeschaltete Verdickungen.

Fig. 3. u. 3 A-C. Motorische Nervenendigung an Muskelfäden des Menschen ohne ausgeprägte Endplattenbildung. 3 mit Seibert Immers. VII/O. $3 \mathrm{~A}-\mathrm{C}$ mit $\mathrm{VII} / \mathrm{I}$.

a Endanschwellungen der Terminalfasern.

d Intercalare Verdickungen derselben.

Fig. 4. A u. B. Muskelfäden mit Endplatten in Seitenansicht vom Menschen. Seibert Immers. VII/I.

a Endanschwellungen der Terminalfasern.

d Intercalare Verdickungen derselben. 
Ueber d. Endigung d. Nerven im quergestreiften Muskel d. Wirbelthiere. 389

Fig. 5 u. 6. Muskelfäden mit Endplatten in Flächenansicht vom Meerschweinchen. Seibert Immers. VII/I.

a Endanschwellungen der Terminalfasern.

b Zugerundetes Ende derselben.

c Zugespitztes Ende derselben.

d Intercalare Verdickungen.

e Isolirte Verdickungen.

Fig. 7. Muskelfaden mit Endplatte in Seitenansicht vom Meersehweinchen. Seibert Immers. VIr/I.

a Endanschwellungen der Terminalfasern.

b Zugerundetes Ende derselben.

e Isolirte Verdickungen.

Fig. 8, $9 \mathrm{~A}$ u. B. Muskelfäden mit Endplatten in Flächenansicht rom Hund. Seibert Immers. VII/O.

a Endanschwellungen.

d Intercalare Verdickungen.

Fig. 10. Muskelfaden mit Endplatte in Seitenansicht vom Hund. Seibert Immers. VII/O.

e Isolirte Verdickungen.

Fig. 11. Muskelfaden mit Endplatte in Flächenansicht vom Schwein. Seibert Immers. VII/I.

a Endanschwellungen.

d Intercalare Verdickung.

c Isolirte Verdickungen.

Fig. 11 A u. B. Muskelfäden mit Eudplatten in Flächenansicht vom Kaninchen. Seibert Immers. VII/I.

a Endanschwellungen.

d Intercalare Verdickungen.

e Isolirte Verdickungen.

Fig. 11 C. Muskelfaden mit Endplatte in Flächenansicht von der Katze. Seibert Immers. VII/I.

a Wahrscheinlich Endanschwellung.

(Der Zusammenhang der goldgefärbten Fasern nicht erhalten, weswegen sie isolirte Verdickungen darstellen.)

Fig. 12. Muskelfaden mit Endplatte in Flächenansicht von der Ente. Seibert. Immers. VII/I.

a. Endanschwellungen.

Fig. 13 u. 14. Muskelfäden mit Endplatten in Flächen- und theilweise auch in Seitenansicht von der Taube. Seibert. Immers. VII/I.

a Endanschwellungen.

Fig. 15. Muskelfaser mit Endplatte in Flächenansicht von Lacerta viridis (Oberarmmuskel) Seibert Immers. VII/I. 
390 E. Fischer: Endigung d. Nerven im quergestreiften Muskel d. Wirbelthiere.

Fig. 16. Muskelfaden mit Endplatte in Seitenansicht aus einem OberschenkelMuskel desselben Thieres. Seibert Immers. VII/III. (Muskelstructur nach Immers. VIr/III eingezeichnet.)

Fig. 17. Stück einer vergoldeten Muskelfaser von demselben Thiere. Seibert Immers. VII/II.

Fig. 18. Muskelfaden mit der Nervenendigung vom Frosch. Seibert Immersion VIr/r. (Die Fasern d und e sind verkürzt gezeichnet.)

Fig. 19. Motorische Nervenendigung vom Frosch, durch Druck vom contraktilen Inhalt getrennt. Seibert Immers. VII/0.

a Kerne d. Terminalfasern von der Fläche,

b dieselben von der Seite gesehen.

Fig. 20. Muskelfasern mit Nervenendigung vom Frosch. Cohnheim'sche Methode. Seibert Immers. VII/I.

Fig. 21. Muskelfasern mit Nervenendigung vom Frosch (Oberarm-Muskel). Gerlach'sche Methode. gl. Vergr.

Fig. 22. Goldgefärbte Streifen aus einem Muskelfaden des Frosches. Unregelmässiger, völlig gebogener Verlauf derselben, in Folge dessen dieselben netzförmig zusammen zu hängen scheinen. Seibert. Immers. VII/I.

Fig. 23. Muskelfaden des Frosches mit Sprenkelungen, gl. Vergr.

Fig. 24. Muskelfaden des Frosches, an welchem die Sprenkelungen (a) in parallele Längsstreifen (b) übergehen, gl. Vergr.

Fig. 25. Stück einer Muskelfaser des Frosches. Beziehung der goldgef. Körperchen zu d. Querstr. Seibert Immers. VII/I.

Fig. 26. Stück einer Muskelfaser des Meerschweinchens. Seibert Immers. VII/I.

Fig. 27. Nervenfasern aus dem Herzen des Hundes. Nur die in einer Einstellungsebene gelegenen Fasern sind gezeichnet, um die Dichtigkeit der Lage derselben zu zeigen. Muskelfasern weggelassen. Hartnack $\mathrm{VIII} / 3$.

Fig. 28. Netzförmig zusammenhängende Nervenfasern gröberen Calibers, ebendaher. Hartnack VIII/3.

a Scheinbar feines Ende einer Nervenfaser.

b Intensiv geschwärzte Stelle einer Nervenfaser.

c Intercalare und intensiv geschwärzte Anschwellungen.

Fig. 29. Netzförmig zusammenhängende Nervenfasern feinsten Calibers, ebendaher. Seibert Immers. VII/I.

Fig. 30. Querschnitte der vergoldeten Muskelmasse des Herzens. Seibert Immers. VII/I.

Fig. 31. Verästelung einer dicken Nervenfaser. Uebergang in die der Längsrichtung der Muskelfäden parallel laufenden Nervenfasern, die späterhin netzförmig zusammenhängen. Oberhäuser Syst. 4/0k.3.

Fig. 32. Muskelfäden des Herzens vom Hund, durch lokalen Goldniederschlag geschwärzt. Seibert Immers. VIr/I. 


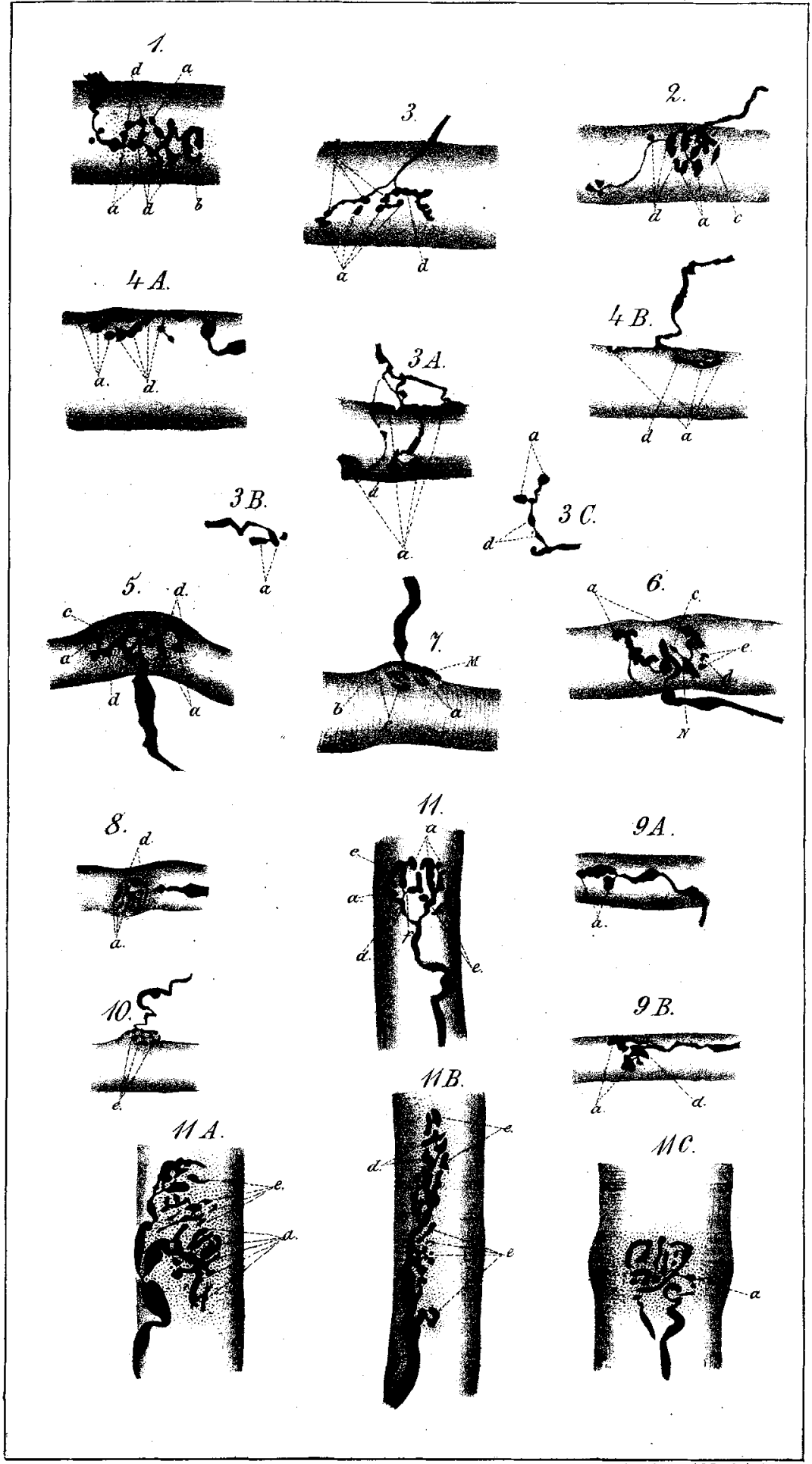




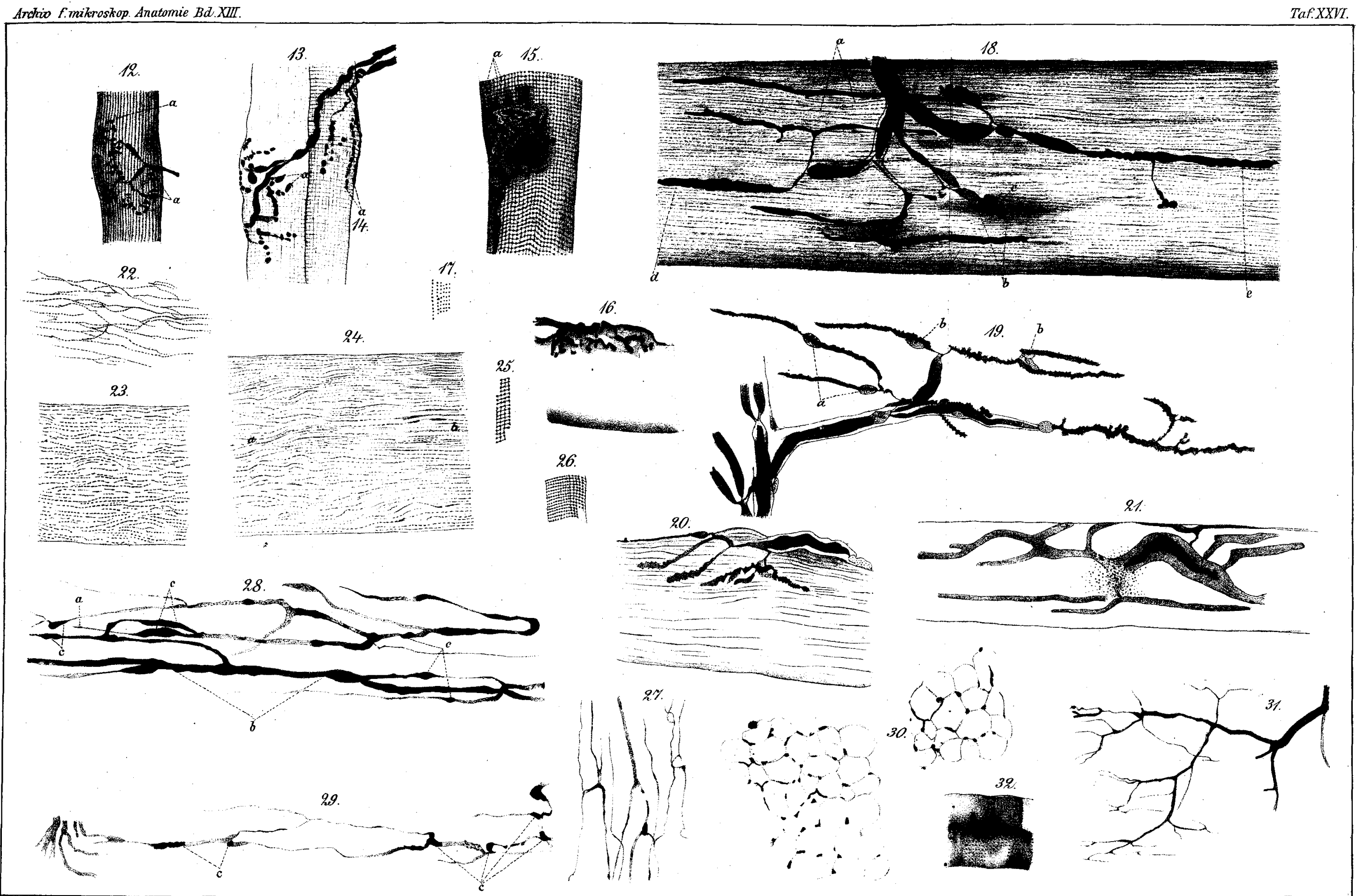

